\title{
DEFORMATION INVARIANCE OF PLURIGENERA
}

\author{
HAJIME TSUJI
}

\begin{abstract}
We prove the invariance of plurigenera under smooth projective deformations in full generality.
\end{abstract}

\section{$\S 1$. Introduction}

Let $X$ be a smooth projective variety and let $K_{X}$ be the canonical bundle of $X$. The canonical ring

$$
R\left(X, K_{X}\right):=\oplus_{m \geq 0} H^{0}\left(X, \mathcal{O}_{X}\left(m K_{X}\right)\right)
$$

is a basic birational invariant of $X$. For every positive integer $m$, the $m$-th plurigenus $P_{m}(X)$ is defined by

$$
P_{m}(X):=\operatorname{dim} H^{0}\left(X, \mathcal{O}_{X}\left(m K_{X}\right)\right) .
$$

The plurigenera are fundamental birational invariants of compact complex manifolds. It has been believed that the plurigenera is invariant under smooth projective deformations (although the plurigenera are not deformation invariant under nonprojective deformations $([9])$ ).

In this paper, we shall prove the invariance of plurigenera under smooth projective deformations in full generality.

TheOREm 1.1. Let $\pi: X \longrightarrow \Delta$ be a smooth projective family of smooth projective varieties over the unit open disk.

Then for every positive integer $m$, the $m$-th plurigenus $P_{m}\left(X_{t}\right)\left(X_{t}:=\right.$ $\left.\pi^{-1}(t)\right)$ is independent of $t \in \Delta$.

In the course of the proof of Theorem 1.1, we also prove the following theorem.

Received February 8, 2001.

2000 Mathematics Subject Classification: 32J25. 
Theorem 1.2. Let $\pi: X \longrightarrow \Delta$ be a smooth projective family. Suppose that $K_{X_{0}}$ is pseudoeffective. Then $K_{X_{t}}$ is pseudoeffective for every $t \in \Delta$.

Let us recall the former results concerning Theorem 1.1. S. Iitaka proved the invariance of plurigenera for deformations of compact complex surfaces ([3]) by using the classification of compact complex surfaces. N. Nakayama ([10]) pointed out that the invariance of plurigenera for smooth projective deformations can be derived if the minimal model program were completed for families (it is worth noting that his results (including the lowersemicontinuity of plurigenera under semistable degenerations) immediately follow from the $L^{2}$-extension theorem ([13, p.200, Theorem]) without using Kollár's vanishing theorem $([5]))$.

Recently Y.-T. Siu ([14]) proved that for every $m \geq 1, P_{m}(X)$ is invariant under smooth projective deformations, if all the fibers are of general type. The proof of [14] has been translated into more algebraic way and the result in [14] has been slightly generalized by [4, 11] (although such translation is completely unnecessary for such generalizations).

For the proof of Theorem 1.1, the central problem is the existence of singular hermitian metric $h$ on $K_{X}$ such that the curvature current $\Theta_{h}$ is semipositive and $\left.h\right|_{X_{t}}$ is an AZD (cf. Definition 2.3) of $K_{X_{t}}$ for every $t \in \Delta$. As soon as we construct such a metric $h$, the $L^{2}$-extension theorem ([13]) implies the invariance of the plurigenera. Here the key point is that the $L^{2}$-extension theorem requires the semipositivity of the curvature of the singular hermitian metric, but it does not require any strict positivity of the curvature. In this sense the $L^{2}$-extension theorem is similar to Kollár's vanishing theorem $([5])$ in algebraic context.

The construction of the above singular hermitian metric $h$ consists of the inductive estimates of singular hermitian metrics using Bergman kernels. This is more straightforward than the inductive comparison of multiplier ideal sheaves as in [14, p. 670, Proposition 5]. In this sense the proof is quantitative and not qualitative. The essential idea is the dynamical construction of an AZD of the canonical line bundle of a smooth projective variety with pseudoeffective canonical line bundle. This construction works only for pseudoeffective canonical line bundles. This clarifies why the canonical line bundle is special. Here the key ingredient of the proof is the $L^{2}$-extension theorem of holomorphic sections $[13,8,12]$. And we use the fact that the operator norm of the interpolation operator is bounded from 
above by a universal constant.

Hence the proof here is analytic in nature. The advantage of the $L^{2}$ estimates of $\bar{\partial}$-operators is that it assures the existence of solutions of $\bar{\partial}$ equations with estimates, while the algebraic methods only indicate the existence of solutions. This is the main reason why the $L^{2}$-estimates changed the complex analysis completely.

Another key of the proof of Theorem 1.1 is the theory of AZD. The theory of AZD can be viewed as a minimal model theory of pseudoeffective line bundles. The theory of AZD is very simple (cf. Theorem 2.2 ) and fits to the $L^{2}$-extension theorem and many other analytic tools. As in $[14,11,4]$, we may replace AZD's with some infinite sequence of multiplier ideal sheaves, if the line bundle is big. But this kind of translation breaks down when the line bundle is not big. This is because in general a singular hermitian metric with semipositive curvature current cannot be approximated by a sequence of singular hermitian metrics with algebraic singularities and semipositive curvature, while singular hermitian metrics with strictly positive curvature current can be approximated by a sequence of singular hermitian metric with algebraic singularities and semipositive curvature current ([1]).

The author would like to express his hearty thanks to Professor $\mathrm{T}$. Ohsawa for his interest in this work and his encouragement.

\section{§2. Preliminaries}

\subsection{Multiplier ideal sheaves}

In this subsection $L$ will denote a holomorphic line bundle on a complex manifold $M$.

Definition 2.1. A singular hermitian metric $h$ on $L$ is given by

$$
h=e^{-\varphi} \cdot h_{0}
$$

where $h_{0}$ is a $C^{\infty}$-hermitian metric on $L$ and $\varphi \in L_{l o c}^{1}(M)$ is an arbitrary function on $M$. We call $\varphi$ a weight function of $h$.

The curvature current $\Theta_{h}$ of the singular hermitian line bundle $(L, h)$ is defined by

$$
\Theta_{h}:=\Theta_{h_{0}}+\sqrt{-1} \partial \bar{\partial} \varphi
$$


where $\partial \bar{\partial}$ is taken in the sense of a current. The $L^{2}$-sheaf $\mathcal{L}^{2}(L, h)$ of the singular hermitian line bundle $(L, h)$ is defined by

$$
\mathcal{L}^{2}(L, h):=\left\{\sigma \in \Gamma\left(U, \mathcal{O}_{M}(L)\right) \mid h(\sigma, \sigma) \in L_{l o c}^{1}(U)\right\}
$$

where $U$ runs over the open subsets of $M$. In this case there exists an ideal sheaf $\mathcal{I}(h)$ such that

$$
\mathcal{L}^{2}(L, h)=\mathcal{O}_{M}(L) \otimes \mathcal{I}(h)
$$

holds. We call $\mathcal{I}(h)$ the multiplier ideal sheaf of $(L, h)$. If we write $h$ as

$$
h=e^{-\varphi} \cdot h_{0}
$$

where $h_{0}$ is a $C^{\infty}$ hermitian metric on $L$ and $\varphi \in L_{l o c}^{1}(M)$ is the weight function, we see that

$$
\mathcal{I}(h)=\mathcal{L}^{2}\left(\mathcal{O}_{M}, e^{-\varphi}\right)
$$

holds. For $\varphi \in L_{l o c}^{1}(M)$ we define the multiplier ideal sheaf of $\varphi$ by

$$
\mathcal{I}(\varphi):=\mathcal{L}^{2}\left(\mathcal{O}_{M}, e^{-\varphi}\right)
$$

Similarly we define

$$
\mathcal{I}_{\infty}(h):=\mathcal{L}^{\infty}\left(\mathcal{O}_{M}, e^{-\varphi}\right)
$$

and call it the $L^{\infty}$-multiplier ideal sheaf of $(L, h)$.

Definition 2.2. $L$ is said to be pseudoeffective, if there exists a singular hermitian metric $h$ on $L$ such that the curvature current $\Theta_{h}$ is a closed positive current.

Also a singular hermitian line bundle $(L, h)$ is said to be pseudoeffective, if the curvature current $\Theta_{h}$ is a closed positive current.

\subsection{Analytic Zariski decompositions}

Definition 2.3. Let $M$ be a compact complex manifold and let $L$ be a holomorphic line bundle on $M$. A singular hermitian metric $h$ on $L$ is said to be an analytic Zariski decomposition, if the followings hold.

1. $\Theta_{h}$ is a closed positive current,

2. for every $m \geq 0$, the natural inclusion

$$
H^{0}\left(M, \mathcal{O}_{M}(m L) \otimes \mathcal{I}\left(h^{m}\right)\right) \rightarrow H^{0}\left(M, \mathcal{O}_{M}(m L)\right)
$$

is an isomorphism. 
Remark 2.1. If an AZD exists on a line bundle $L$ on a smooth projective variety $M, L$ is pseudoeffective by the condition 1 above.

THEOREM 2.1. ([15, 16]) Let $L$ be a big line bundle on a smooth projective variety $M$. Then $L$ has an $A Z D$.

As for the existence for general pseudoeffective line bundles, now we have the following theorem.

TheOREM 2.2. ([2, Theorem 1.5]) Let $X$ be a smooth projective variety and let $L$ be a pseudoeffective line bundle on $X$. Then $L$ has an AZD.

Proof of Theorem 2.2. Although the proof is in [2], we shall give a proof here, because we shall use it afterwards.

Let $h_{0}$ be a fixed $C^{\infty}$-hermitian metric on $L$. Let $E$ be the set of singular hermitian metric on $L$ defined by

$E=\{h ; h:$ lowersemicontinuous singular hermitian metric on $L$,

$$
\left.\Theta_{h} \text { is positive, } \frac{h}{h_{0}} \geq 1\right\} \text {. }
$$

Since $L$ is pseudoeffective, $E$ is nonempty. We set

$$
h_{L}=h_{0} \cdot \inf _{h \in E} \frac{h}{h_{0}},
$$

where the infimum is taken pointwise. The supremum of a family of plurisubharmonic functions uniformly bounded from above is known to be again plurisubharmonic, if we modify the supremum on a set of measure 0(i.e., if we take the uppersemicontinuous envelope) by the following theorem of $\mathrm{P}$. Lelong.

TheOREM 2.3. ([7, p.26, Theorem 5]) Let $\left\{\varphi_{t}\right\}_{t \in T}$ be a family of plurisubharmonic functions on a domain $\Omega$ which is uniformly bounded from above on every compact subset of $\Omega$. Then $\psi=\sup _{t \in T} \varphi_{t}$ has a minimum uppersemicontinuous majorant $\psi^{*}$ which is plurisubharmonic. We call $\psi^{*}$ the uppersemicontinuous envelope of $\psi$.

Remark 2.2. In the above theorem the equality $\psi=\psi^{*}$ holds outside of a set of measure 0 (cf.[7, p.29]). 
By Theorem 2.3, we see that $h_{L}$ is also a singular hermitian metric on $L$ with $\Theta_{h} \geq 0$. Suppose that there exists a nontrivial section $\sigma \in$ $\Gamma\left(X, \mathcal{O}_{X}(m L)\right)$ for some $m$ (otherwise the second condition in Definition 2.3 is empty). We note that

$$
\log |\sigma| \frac{2}{m}
$$

gives the weight of a singular hermitian metric on $L$ with curvature $2 \pi m^{-1}(\sigma)$, where $(\sigma)$ is the current of integration along the zero set of $\sigma$. By the construction we see that there exists a positive constant $c$ such that

$$
\frac{h_{0}}{|\sigma|^{\frac{2}{m}}} \geq c \cdot h_{L}
$$

holds. Hence

$$
\sigma \in H^{0}\left(X, \mathcal{O}_{X}(m L) \otimes \mathcal{I}_{\infty}\left(h_{L}^{m}\right)\right)
$$

holds. Hence in particular

$$
\sigma \in H^{0}\left(X, \mathcal{O}_{X}(m L) \otimes \mathcal{I}\left(h_{L}^{m}\right)\right)
$$

holds. This means that $h_{L}$ is an AZD of $L$.

Remark 2.3. By the above proof we have that for the AZD $h_{L}$ constructed as above

$$
H^{0}\left(X, \mathcal{O}_{X}(m L) \otimes \mathcal{I}_{\infty}\left(h_{L}^{m}\right)\right) \simeq H^{0}\left(X, \mathcal{O}_{X}(m L)\right)
$$

holds for every $m$.

It is easy to see that the multiplier ideal sheaves of $h_{L}^{m}(m \geq 1)$ constructed in the proof of Theorem 2.2 are independent of the choice of the $C^{\infty}$-hermitian metric $h_{0}$. We call the AZD constructed as in the proof of Theorem 2.2 a canonical AZD of $L$.

\section{3. $\quad L^{2}$-extension theorem}

The following theorem is crucial in our proof of Theorem 1.1.

Theorem 2.4. ([13, p.200, Theorem $])$ Let $X$ be a Stein manifold of dimension $n, \psi$ a plurisubharmonic function on $X$ and $s$ a holomorphic function on $X$ such that $d s \neq 0$ on every branch of $s^{-1}(0)$. We put $Y:=$ 
$s^{-1}(0)$ and $Y_{0}:=\{x \in Y ; d s(x) \neq 0\}$. Let $g$ be a holomorphic $(n-1)$-form on $Y_{0}$ with

$$
c_{n-1} \int_{Y_{0}} e^{-\psi} g \wedge \bar{g}<\infty,
$$

where $c_{k}=(-1)^{\frac{k(k-1)}{2}}(\sqrt{-1})^{k}$. Then there exists a holomorphic $n$-form $G$ on $X$ such that

$$
G(x)=g(x) \wedge d s(x)
$$

on $Y_{0}$ and

$$
c_{n} \int_{X} e^{-\psi}\left(1+|s|^{2}\right)^{-2} G \wedge \bar{G} \leq 1620 \pi c_{n-1} \int_{Y_{0}} e^{-\psi} g \wedge \bar{g} .
$$

\section{§3. Dynamical construction of an AZD}

Let $X$ be a smooth projective variety and let $K_{X}$ be the canonical line bundle of $X$. Let $n$ denote the dimension of $X$.

Let $A$ be a sufficiently ample line bundle on $X$ such that for every pseudoeffective singular hermitian line bundle $\left(L, h_{L}\right)$

$$
\mathcal{O}_{X}(A+L) \otimes \mathcal{I}\left(h_{L}\right)
$$

and

$$
\mathcal{O}_{X}\left(K_{X}+A+L\right) \otimes \mathcal{I}\left(h_{L}\right)
$$

are globally generated. This is possible by [14, p. 667, Proposition 1]. Let $h_{A}$ be a $C^{\infty}$ hermitian metric on $A$ with strictly positive curvature.

Hereafter we shall assume that $\kappa(X) \geq 0$, in particular $K_{X}$ is pseudoeffective. For $m \geq 0$, let $h_{m}$ be the singular hermitian metrics on $A+m K_{X}$ constructed as follows. Let $h_{0}$ be a $C^{\infty}$-hermitian metric $h_{A}$ on $A$ with strictly positive curvature. Suppose that $h_{m-1}(m \geq 1)$ has been constructed. Let $\left\{\sigma_{0}^{(m)}, \ldots, \sigma_{N(m)}^{(m)}\right\}$ be an orthonormal basis of $V_{m}:=$ $H^{0}\left(X, \mathcal{O}_{X}\left(A+m K_{X}\right) \otimes \mathcal{I}\left(h^{m-1}\right)\right)$ with respect to the inner product :

$$
\begin{aligned}
\left(\sigma, \sigma^{\prime}\right) & :=\int_{X} \sigma \cdot \overline{\sigma^{\prime}} \cdot h_{m-1} \\
& =\int_{X} \sigma \cdot \overline{\sigma^{\prime}} \cdot\left(h_{m-1} \otimes(d V)^{-1}\right) \cdot d V,
\end{aligned}
$$

where $d V$ is an arbitrary nowhere degenerate $C^{\infty}$ volume form on $X$. We set

$$
K_{m}:=\sum_{i=0}^{N(m)}\left|\sigma_{i}^{(m)}\right|^{2}
$$


where $\left|\sigma_{i}^{(m)}\right|^{2}$ denotes $\sigma_{i}^{(m)} \cdot \overline{\sigma_{i}^{(m)}}$. We call $K_{m}$ the Bergman kernel of $A+m K_{X}$ with respect to $h_{m-1} \otimes(d V)^{-1}$ and $d V$. Clearly it is independent of the choice of the orthonormal basis. We define the integer $\nu$ by

$$
\nu:=\varlimsup_{m \rightarrow \infty} \frac{\log \operatorname{dim} H^{0}\left(X, \mathcal{O}_{X}\left(A+m K_{X}\right) \otimes \mathcal{I}\left(h^{m}\right)\right)}{\log m} .
$$

And we define the singular hermitian metric $h_{m}$ on $A+m K_{X}$ by

$$
h_{m}:=m^{\nu} \cdot K_{m}^{-1} .
$$

It is clear that $K_{m}$ has semipositive curvature in the sense of currents. We note that for every $x \in X$

$$
K_{m}(x)=\sup \left\{|\sigma|^{2}(x) ; \sigma \in V_{m}, \int_{X} h_{m-1} \cdot|\sigma|^{2}=1\right\}
$$

holds by definition (cf. [6, p.46, Proposition 1.4.16]).

Let $d V$ be a $C^{\infty}$-volume form on $X$ with respect to a Kähler form $\omega$ on $X$. For a singular hermitian line bundle $\left(F, h_{F}\right)$ on $X$, let $A^{2}\left(M, F, h_{F}, d V\right)$ denote the Hilbert space of $L^{2}$ holomorphic sections of $F$ with respect to $h_{F}$ and $d V$. We may also assume that for any pseudoeffective singular hermitian line bundle $\left(L, h_{L}\right)$ and for any point $x \in X$, there exists an interpolation operator

$I_{x}: A^{2}\left(x, K_{X} \otimes A \otimes L, d V^{-1} h_{A} h_{L}, \delta_{x}\right) \longrightarrow A^{2}\left(X, K_{X} \otimes A \otimes L, d V^{-1} h_{A} h_{L}, d V\right)$

such that the operator norm of $I_{x}$ is bounded from above by a positive constant independent of $x \in X$ and $\left(L, h_{L}\right)$, where $\delta_{x}$ denotes the Dirac measure at $x$. This is certainly possible, if we take $A$ to be sufficiently ample.

In fact let $x$ be a point on $X$ and let $\left(U, z_{1}, \ldots, z_{n}\right)$ be a local coordinate neighbourhood of $x$ which is biholomorphic to $\Delta^{n}$ and $z_{i}(x)=0(1 \leq i \leq n)$. Then by the successive use of Theorem 2.4, we find an interpolation operator $I_{x}^{U}: A^{2}\left(x, K_{X} \otimes A \otimes L, d V^{-1} h_{A} h_{L}, \delta_{x}\right) \longrightarrow A^{2}\left(U, K_{X} \otimes A \otimes L, d V^{-1} h_{A} h_{L}, d V\right)$

such that the operator norm of $I_{x}^{U}$ is bounded from above by a positive constant $C_{U}$ independent of $\left(L, h_{L}\right)$. Now we note that the curvature of $h_{A} h_{L}$ is bounded from below by the Kähler form $\Theta_{A}$. Let $\rho$ be a $C^{\infty}$-function on $X$ such that Supp $\rho \subset \subset U, 0 \leq \rho \leq 1$ and $\rho \equiv 1$ on a neighbourhood of $x$. 
Let $\sigma_{x}$ be an element of $A^{2}\left(x, K_{X} \otimes A \otimes L, d V^{-1} h_{A} h_{L}, \delta_{x}\right)$. Then replacing $\left(A, h_{A}\right)$ by its sufficiently high positive multiple, we may assume that

$$
\Theta_{A}+(n+1) \sqrt{-1} \partial \bar{\partial}\left(\rho \cdot \log \sum_{i=1}^{n}\left|z_{i}\right|^{2}\right) \geq \omega
$$

holds on $X$. We also note that there exists a positive constant $C_{U}^{\prime}$ independent of $\left(L, h_{L}\right)$ and $\sigma_{x}$ such that

$$
\begin{aligned}
\int_{X} \exp \left(-(n+1) \rho \cdot \log \sum_{i=1}^{n}\left|z_{i}\right|^{2}\right) \cdot & \left|\bar{\partial}\left(\rho \cdot I_{x}^{U} \sigma_{x}\right)\right|^{2} d V \\
\leq & C_{U}^{\prime} \cdot\left(d V^{-1} h_{A} h_{L}\right)\left(\sigma_{x}, \sigma_{x}\right)
\end{aligned}
$$

holds, where $\left|\bar{\partial}\left(\rho \cdot I_{x}^{U} \sigma_{x}\right)\right|^{2}$ denotes the norm with respect to $h_{A} \cdot h_{L}$ and $\omega$. In fact $C_{U}^{\prime}$ only depends on $C_{U}$ and the supremum of the norm of $\bar{\partial} \rho$ with respect to $\omega$. Then by the usual $L^{2}$-estimate, we may assume that we can solve the $\bar{\partial}$-equation

$$
\bar{\partial} u=\bar{\partial}\left(\rho \cdot I_{x}^{U} \sigma_{x}\right)
$$

with

$$
u(x)=0
$$

so that

$$
\int_{X} h_{A} h_{L}|u|^{2} \leq C \cdot\left(d V^{-1} h_{A} h_{L}\right)\left(\sigma_{x}, \sigma_{x}\right)
$$

holds for a positive constant $C$ independent of $\left(L, h_{L}\right)$ and $\sigma_{x}$. Then

$$
\rho \cdot I_{x}^{U} \sigma_{x}-u \in H^{0}\left(X, \mathcal{O}_{X}\left(K_{X}+A+L\right) \otimes \mathcal{I}\left(h_{L}\right)\right)
$$

is an extension of $\sigma_{x}$. Since $X$ is compact, moving $x$ and $U$, by the above estimates this implies the assertion.

LEMma 3.1. Let $h$ be a canonical AZD of $K_{X}$ constructed as in the proof of Theorem 2.2. Then the inclusion:

$$
\mathcal{I}\left(h^{m}\right) \subseteq \mathcal{I}\left(h_{m}\right)
$$

holds for every $m \geq 0$. 
Proof. We prove this lemma by induction on $m$. If $m=0$, then both sides are $\mathcal{O}_{X}$.

Suppose that the inclusion

$$
\mathcal{I}\left(h^{m-1}\right) \subseteq \mathcal{I}\left(h_{m-1}\right)
$$

has been settled for some $m \geq 1$. Then we have that by the property of $A$ as above

$$
\mathcal{O}_{X}\left(K_{X}+\left(A+(m-1) K_{X}\right)\right) \otimes \mathcal{I}\left(h^{m-1}\right)
$$

is a globally generated subsheaf of

$$
\mathcal{O}_{X}\left(A+m K_{X}\right) \otimes \mathcal{I}\left(h_{m-1}\right)
$$

Hence by the definition of $\mathcal{I}\left(h_{m}\right)$ we see that

$$
\mathcal{I}\left(h^{m-1}\right) \subseteq \mathcal{I}\left(h_{m}\right)
$$

holds. In particular

$$
\mathcal{I}\left(h^{m}\right) \subseteq \mathcal{I}\left(h_{m}\right)
$$

holds. By the induction on $m$, this completes the proof of Lemma 3.1.

By the choice of $A$ and Lemma $3.1, h_{m}$ is well defined for every $m \geq 0$. Now we shall make the above lemma quantitative.

LEMma 3.2. There exists a positive constant $C$ such that

$$
h_{m} \leq C^{m} \cdot h_{A} \cdot h^{m}
$$

holds for every $m \geq 0$.

Proof. First if $m=0$, both sides are $h_{0}$. Suppose that for some $m \geq 1$,

$$
h_{m-1} \leq C_{(m-1)} \cdot h_{A} \cdot h^{m-1}
$$

holds for some positive constant $C_{(m-1)}$. Let $d V$ be a $C^{\infty}$ volume form on $X$. Let $C(1)$ be a positive constant such that

$$
h \geq C(1) \cdot(d V)^{-1}
$$

holds on $X$. Let us denote the Bergman kernel of $V_{m}$ with respect to a singular hermitian metric $H$ on $A+m K_{X}$ and the volume form $d V$ by $K(A+$ 
$\left.m K_{X}, H, d V\right)$. In this notation $K_{m}$ is expressed as $K\left(A+m K_{X}, h_{m-1} \otimes\right.$ $\left.(d V)^{-1}, d V\right)$.

Then we have that

$$
\begin{aligned}
& K\left(A+m K_{X}, h_{m-1} \otimes(d V)^{-1}, d V\right) \\
\geq & C_{(m-1)}^{-1} \cdot K\left(A+m K_{X}, h_{A} \cdot h^{m-1} \cdot(d V)^{-1}, d V\right) \\
\geq & C_{(m-1)}^{-1} \cdot C(2) \cdot\left(h_{A} \cdot h^{m-1} \cdot(d V)^{-1}\right)^{-1} \cdot m^{\nu} \\
\geq & C_{(m-1)}^{-1} \cdot C(1) \cdot C(2) \cdot\left(h_{A} \cdot h^{m}\right)^{-1} \cdot m^{\nu}
\end{aligned}
$$

hold for some positive constant $C(2)$. The first inequality follow from the formula :

$$
\begin{aligned}
& K\left(A+m K_{X}, h_{m-1} \otimes(d V)^{-1}, d V\right)(x) \\
& \quad=\sup \left\{|\sigma|^{2}(x) ; \sigma \in V_{m} ; \int_{X} h_{m-1}|\sigma|^{2}=1\right\}(x \in X)
\end{aligned}
$$

and the similar formula for $K\left(A+m K_{X}, h_{A} \cdot h^{m-1} \cdot(d V)^{-1}, d V\right)$.

The 2-nd inequality follows from the $L^{2}$-extension theorem (Theorem 2.4) applied to the extension from a point to $X$ as above. We note that for every point $x \in X$ and every general $\nu$-dimensional subvariety $Y \ni$ $x,\left.\left(K_{x}, h\right)\right|_{Y}$ is big, hence $\left.h\right|_{Y}$ is dominated by a metric with strictly positive curvature. The factor $m^{\nu}$ comes form the fact that the norm of the local interpolation $I_{x}^{U}$ as above is $O\left(m^{-\nu}\right)$ (cf. $[17$, p.105, (1.11)]). Hence we may assume that $C(2)$ is independent of $m$. Now we can take $C$ to be the constant $C(1)^{-1} \cdot C(2)^{-1}$. This completes the proof of Lemma 3.2.

LEMMA 3.3. There exists a positive constant $\tilde{C}$ such that for every $m \geq 1$,

$$
h_{A} \cdot K_{m} \leq \tilde{C}^{m}(d V)^{m} \cdot m^{\nu}
$$

holds.

Proof. Let $p \in X$ be an arbitrary point. Let $\left(U, z_{1}, \ldots, z_{n}\right)$ be a local coordinate around $x$ such that

1. $z_{1}(p)=\cdots=z_{n}(p)=0$,

2. $U$ is biholomorphic to the open unit polydisk in $\mathbf{C}^{n}$ with center $O \in$ $\mathbf{C}^{n}$ by the coordinate,

3. $z_{1}, \ldots, z_{n}$ are holomorphic on a neighbourhood of the closure of $U$, 
4. there exists a holomorphic frame e of $A$ on the closure of $U$.

We set

$$
\Omega:=(-1)^{\frac{n(n-1)}{2}}(\sqrt{-1})^{n} d z_{1} \wedge \cdots \wedge d z_{n} \wedge d \bar{z}_{1} \wedge \cdots \wedge d \bar{z}_{n}
$$

For every $m \geq 0$, we set

$$
C_{m}:=\sup _{x \in U} \frac{h_{A} \cdot K_{m}}{\Omega^{m}} .
$$

We note that for any $x \in X$

$$
K_{m}(x)=\sup \left\{|\phi|^{2}(x) ; \phi \in V_{m}, \int_{X} h_{m-1}|\phi|^{2}=1\right\}
$$

holds. Let $\phi_{0}$ be the element of $\Gamma\left(X, \mathcal{O}_{X}\left(A+m K_{X}\right)\right)$ such that

$$
K_{m}(x)=\left|\phi_{0}\right|^{2}(x)
$$

and

$$
\int_{X} h_{m-1}\left|\phi_{0}\right|^{2}=1
$$

Then there exists a holomorphic function $f$ on $U$ such that

$$
\left.\phi_{0}\right|_{U}=f \cdot\left(d z_{1} \wedge \cdots d z_{n}\right)^{m} \cdot \mathbf{e}
$$

holds. Then

$$
\int_{U} h_{A}\left|\phi_{0}\right|^{2} \Omega^{-(m-1)}=\int_{U}|f|^{2} h_{A}(\mathbf{e}, \mathbf{e}) \Omega
$$

holds. On the other hand by the definition of $C_{m-1}$ we see that

$$
\int_{U} h_{A}\left|\phi_{0}\right|^{2} \Omega^{-(m-1)} \leq C_{m-1} \int_{U} h_{m-1}\left|\phi_{0}\right|^{2} \leq C_{m-1}
$$

hold. Combining above inequalities we have that

$$
\int_{U}|f|^{2} h_{A}(\mathbf{e}, \mathbf{e}) \Omega \leq C_{m-1}
$$

holds. Let $0<\delta<<1$ be a sufficiently small number. Let $U_{\delta}$ be the inverse image of

$$
\left\{\left(y_{1}, \ldots, y_{n}\right) \in \mathbf{C}^{n} \| y_{i} \mid<1-\delta\right\}
$$


by the coordinate $\left(z_{1}, \ldots, z_{n}\right)$.

Then by the subharmonicity of $|f|^{2}$, there exists a positive constant $C_{\delta}$ independent of $m$ such that

$$
|f(x)|^{2} \leq C_{\delta} \cdot C_{m-1}
$$

holds for every $x \in U_{\delta}$. Then we have that

$$
K_{m}(x) \leq C_{\delta} \cdot C_{m-1} \cdot|\mathbf{e}|^{2} \otimes \Omega^{m}(x)
$$

holds for every $x \in U_{\delta}$. Summing up the estimates for orthonormal basis, moving $p$, by the compactness of $X$ we see that there exists a positive constant $\tilde{C}$ such that

$$
h_{A} \cdot K_{m} \leq \tilde{C}^{m} \cdot(d V)^{m} \cdot m^{\nu}
$$

holds on $X$. This completes the proof of Lemma 3.3.

By Lemma 3.2 and Lemma 3.3

$$
K_{\infty}:=\text { the uppersemicontinuous envelope of } \limsup _{m \rightarrow \infty} \sqrt[m]{K_{m}}
$$

is a well defined volume form on $X$ which does not vanish outside of a set of measure 0 . We set

$$
h_{\infty}:=\frac{1}{K_{\infty}} .
$$

Then by Lemma 3.2, we see that

$$
h_{\infty} \leq C \cdot h
$$

holds. By the definition of $h_{\infty}$, it is clear that the curvature $\Theta_{h_{\infty}}$ is semipositive in the sense of current. Hence by the construction of $h$ (see the proof of Theorem 2.2), we see that there exists a positive constant $C^{\prime}$ such that the opposite estimate:

$$
h_{\infty} \geq C^{\prime} \cdot h
$$

holds.

Hence we have the following theorem.

THEOREM 3.1. Let $h_{\infty}$ be the above singular hermitian metric on $K_{X}$. Then $h_{\infty}$ is an $A Z D$ of $K_{X}$. 


\section{§4. Proof of Theorem 1.1}

Now we shall prove Theorem 1.1. Let $\pi: X \longrightarrow \Delta$ be a smooth projective family of projective varieties as in Theorem 1.1. We set $X_{t}:=$ $\pi^{-1}(t)$. If for every $t \in \Delta, K_{X_{t}}$ is not pseudoeffective, then $P_{m}\left(X_{t}\right)=0$ holds for every $t \in \Delta$ and every $m \geq 1$. Hence in this case there is nothing to prove.

Now we shall assume that for some $t_{0} \in \Delta$, say $t_{0}=0, K_{X_{0}}$ is pseudoeffective. Shrinking $\Delta$, if necessary, we may assume that there exists an ample line bundle $A$ on $X$ such that for any pseudoeffective singular hermitian line bundle $\left(L, h_{L}\right)$

$$
\mathcal{O}_{X}(A+L) \otimes \mathcal{I}\left(h_{L}\right)
$$

and

$$
\mathcal{O}_{X}\left(K_{X}+A+L\right) \otimes \mathcal{I}\left(h_{L}\right)
$$

are globally generated and for every $t \in \Delta$ and for any pseudoeffective singular hermitian line bundle $\left(L_{t}, h_{L_{t}}\right)$ on $X_{t}$,

$$
\mathcal{O}_{X_{t}}\left(\left.A\right|_{X_{t}}+L_{t}\right) \otimes \mathcal{I}\left(h_{L_{t}}\right)
$$

and

$$
\mathcal{O}_{X_{t}}\left(K_{X_{t}}+\left.A\right|_{X_{t}}+L_{t}\right) \otimes \mathcal{I}\left(h_{L_{t}}\right)
$$

are globally generated. Let $h_{A}$ be a $C^{\infty}$-hermitian metric on $A$ such that $\Theta_{h_{A}}$ is a Kähler form on $X$. We set

$$
h_{0}:=h_{A}
$$

and

$$
h_{0, t}:=\left.h_{A}\right|_{X_{t}} \quad(t \in \Delta) .
$$

Let us fix an arbitrary $t \in \Delta$. As in Section 3, inductively we shall define the sequences of singular hermitian metrics $\left\{h_{m}\right\}$ on $X$ and $\left\{h_{m, t}\right\}$ on $X_{t}(t \in$ $\Delta)$. In this case $X$ is noncompact, but using the sections which restricts to $V_{m, t}$ above and the normalization constants $m^{\nu+1}(m \geq 1)$ (here we use the Cauchy estimates on the disk of radius $O(1 \sqrt{m})$ ), the construction works as in Section 3. However we should note that we do not know the pseudoeffectivity of $K_{X}$ or $K_{X_{t}}\left(t \in \Delta^{*}\right)$ apriori. Hence at this stage $h_{m}$ and $h_{m, t}\left(t \in \Delta^{*}\right)$ are not well defined for $m \geq 2$.

But by the $L^{2}$-extension theorem (Theorem 2.4 or [8]) (as in the proof of Lemma 4.1), we have the following lemma. 
LEMmA 4.1. Let $t$ be a point on $\Delta$. Suppose that $h_{m-1}, h_{m-1, t}$ have been defined and

$$
\mathcal{I}\left(h_{m-1, t}\right) \subseteq \mathcal{I}\left(\left.h_{m-1}\right|_{X_{t}}\right)
$$

holds on $X_{t}$. Then every element of

$$
H^{0}\left(X_{t}, \mathcal{O}_{X_{t}}\left(K_{X_{t}}+\left.A\right|_{X_{t}}+(m-1) K_{X_{t}}\right) \otimes \mathcal{I}\left(h_{m-1, t}\right)\right)
$$

extends to an element of

$$
H^{0}\left(X, \mathcal{O}_{X}\left(K_{X}+A+(m-1) K_{X}\right) \otimes \mathcal{I}\left(h_{m-1}\right)\right)
$$

Proof. We note that since Theorem 2.4 is stated for Stein manifolds, we cannot apply it directly. Let $U$ be a Zariski open Stein subset of $X$ such that $X_{t} \cap U$ is nonempty and $\left.K_{X}\right|_{U}$ is holomorphically trivial. Then for every element $\sigma_{t}$ of

$$
H^{0}\left(X_{t}, \mathcal{O}_{X_{t}}\left(K_{X_{t}}+\left.A\right|_{X_{t}}+(m-1) K_{X_{t}}\right) \otimes \mathcal{I}\left(h_{m-1, t}\right)\right)
$$

$\left.\sigma_{t}\right|_{U \cap X_{t}}$ extends to an element $\sigma$ of

$$
H^{0}\left(U, \mathcal{O}_{X}\left(K_{X}+A+(m-1) K_{X}\right) \otimes \mathcal{I}\left(h_{m-1}\right)\right)
$$

with the $L^{2}$-condition

$$
\int_{U} h_{m-1}|\sigma|^{2}<\infty
$$

But this $L^{2}$-condition implies that $\sigma$ extends to a section of

$$
H^{0}\left(X, \mathcal{O}_{X}\left(K_{X}+A+(m-1) K_{X}\right) \otimes \mathcal{I}\left(h_{m-1}\right)\right)
$$

Since $K_{X_{0}}$ is pseudoeffective, using Lemma 4.1 , we have that $h_{m}$ is well defined for every $m \geq 0$ and the inclusion $\mathcal{I}\left(h_{m, 0}\right) \subset \mathcal{I}\left(\left.h_{m}\right|_{X_{0}}\right)$ holds for every $m \geq 0$ inductively. Hence for every $m \geq 1$ we have a proper Zariski closed subset $S_{m}$ in $\Delta$ such that for every $t \in \Delta-S_{m}, h_{m, t}$ is well defined. In particular $K_{X_{t}}$ is pseudoeffective for every $t \in \Delta-\cup_{m \geq 1} S_{m}$. This implies, by Grauert's coherency theorem that $K_{X_{t}}$ is pseudoeffective for every $t \in \Delta$. Then using Lemma 4.1 and Lemma 3.1, by induction on $m$, we have the following lemma. 
Lemma 4.2. $h_{m}, h_{m, t}(t \in \Delta)$ are well defined for every $m \geq 0$ and $t \in \Delta$. And

$$
\mathcal{I}\left(h_{m, t}\right) \subseteq \mathcal{I}\left(h_{m} \mid X_{t}\right)
$$

holds for every $m \geq 0$ and $t \in \Delta$.

We define

$$
h_{\infty}:=\text { the lowersemicontinuous envelope of } \liminf _{m \rightarrow \infty} \sqrt[m]{h_{m}}
$$

and

$$
h_{\infty, t}:=\text { the lowersemicontinuous envelope of } \liminf _{m \rightarrow \infty} \sqrt[m]{h_{m, t}}
$$

Although $X$ is noncompact, the argument in Section 3 is still valid. In fact since $X$ admits a continuous plurisubharmonic exhaustion function, Lemma 3.2 holds in this case, if we restrict the family to a relatively compact subset of $\Delta$. Also Lemma 3.3 is valid on every relatively compact subset of $X$, since the proof is local. Hence $h_{\infty}, h_{\infty, t}$ are well defined AZD's on $K_{X}$ and $K_{X_{t}}$ respectively.

Again by the $L^{2}$-extension theorem (Theorem 2.4), as Lemma 3.2, we have the following lemma.

Lemma 4.3. For every $t$, there exists a positive constant $C$ such that

$$
\left.h_{m}\right|_{X_{t}} \leq C^{m} \cdot h_{m, t}
$$

holds for every $m \geq 0$. In particular

$$
\left.h_{\infty}\right|_{X_{t}} \leq C \cdot h_{\infty, t}
$$

holds.

Here we have used the fact that for every

$$
\sigma_{t} \in A^{2}\left(X_{t}, K_{X_{t}} \otimes A \otimes K_{X}^{\otimes(m-1)},\left.h_{m-1}\right|_{X_{t}}\right),
$$

there exists an element of

$$
\sigma \in A^{2}\left(X, A \otimes K_{X}^{\otimes m}, h_{m-1}\right)
$$

such that $\left.\sigma\right|_{X_{t}}=\sigma_{t}$ and

$$
\|\sigma\| \leq C_{t}\left\|\sigma_{t}\right\|
$$


where $C_{t}$ is a positive constant depending only on $t$ (if $t=0$, we may take $C_{0}$ to be $2 \sqrt{1620 \pi}$ by Theorem 2.4). Here we note that the $L^{2}$-spaces above are determined without specifying volume forms.

Now by Lemma 4.3 and Theorem 3.1, we see that $\left.h_{\infty}\right|_{X_{t}}$ is an AZD of $K_{X_{t}}$. Then by the $L^{2}$-extension theorem (Theorem 2.4 , see also the proof of Lemma 4.1), we see that for every $m \geq 1$, every element of

$$
H^{0}\left(X_{t}, \mathcal{O}_{X_{t}}\left(m K_{X_{t}}\right) \otimes \mathcal{I}\left(\left.h_{\infty}^{m-1}\right|_{X_{t}}\right)\right) \simeq H^{0}\left(X_{t}, \mathcal{O}_{X_{t}}\left(m K_{X_{t}}\right)\right)
$$

extends to an element of

$$
H^{0}\left(X, \mathcal{O}_{X}\left(K_{X}+(m-1) K_{X}\right) \otimes \mathcal{I}\left(h_{\infty}^{m-1}\right)\right) .
$$

Hence we see that $P_{m}\left(X_{t}\right)$ is lowersemicontinuous. By the upper semicontinuity theorem for cohomologies, we see that $P_{m}\left(X_{t}\right)$ is independent of $t \in \Delta$. This completes the proof of Theorem 1.1 .

Theorem 1.2 has already been proved in the course of the above proof.

\section{$\S 5$. Further generalization}

The proof of the following generalization of Theorem 1.1 is completely parallel to that of Theorem 1.1 (see the assumption on $Y$ in Theorem 2.4).

THEOREM 5.1. Let $\pi: X \longrightarrow \Delta$ be a flat projective family. Suppose that $\pi$ is semistable and $X_{0}=\sum_{i \in I} \Gamma_{i}$ is the only singular fiber. Then

$$
\sum_{i \in I} P_{m}\left(\Gamma_{i}\right) \leq P_{m}\left(X_{t}\right)
$$

holds for every $m \geq 1$ and $t \in \Delta^{*}$.

\section{REFERENCES}

[1] J.P. Demailly, Regularization of closed positive currents and intersection theory, J. of Alg. Geom., 1 (1992), 361-409.

[2] J.P. Demailly, T. Peternell and M. Schneider, Pseudo-effective line bundles on compact Kähler manifolds, math. AG/0006025 (2000).

[3] S. Iitaka, Deformations of complex surfaces, II, J. Math. Soc. of Japan, 22 (1970), $247-261$.

[4] Y. Kawamata, Deformations of canonical singularities, J. of Amer. Math. Soc., 12 (1999), 85-92.

[5] J. Kollár, Higher direct images of dualizing sheaves, I, Ann. of Math. (2), 123 (1986), no. $1,11-42$. 
[6] S. Krantz, Function Theory of Several Complex Variables, John Wiley and Sons, 1982.

[7] P. Lelong, Fonctions Plurisousharmoniques et Formes Differentielles Positives, Gordon and Breach, 1968.

[8] L. Manivel, Un theoreme de prolongement $L^{2}$ de sections holomorphes d'un fibre hermitien $\left[A n L^{2}\right.$ extension theorem for the holomorphic sections of a Hermitian bundle], Math. Z., 212 (1993), no. 1, 107-122.

[9] I. Nakamura, Complex parallelisable manifolds and their small deformations, J. Differential Geom., 10 (1975), 85-112.

[10] N. Nakayama, Invariance of plurigenera of algebraic varieties under minimal model conjectures, Topology, 25, 237-251.

[11] Invariance of plurigenera of algebraic varieties, RIMS preprint (1998).

[12] T. Ohsawa, On the extension of $L^{2}$ holomorphic functions $V$, effects of generalization,, Nagoya Math. J., 161 (2001), 1-21.

[13] T. Ohsawa and K. Takegoshi, $L^{2}$-extension of holomorphic functions, Math. Z., 195 (1987), 197-204.

[14] Y.-T. Siu, Invariance of plurigenera, Invent. Math., 134 (1998), 661-673.

[15] H. Tsuji, Analytic Zariski decomposition, Proc. of Japan Acad., 61 (1992), 161-163.

[16] — Existence and Applications of Analytic Zariski Decompositions, Analysis and Geometry in Several Complex Variables (Komatsu and Kuranishi ed.), Trends in Math., 253-271, Birkhäuser, 1999.

[17] G. Tian, On a set of polarized Kähler metrics on algebraic manifolds, J. Differential Geom., 32 (1990), 99-130.

Note added in proofs

For a generalization of Theorem 1.1, see the author's preprint: "Subadjunction theorem for pluricanonical divisors", math.AG/0111311.

Department of Mathematics

Tokyo Institute of Technology

2-12-1 Ohokayama, Megro 152-8551

Japan

tsuji@math.titech.ac.jp 